\title{
Neurological impairment among heterozygote women for X-linked Adrenoleukodystrophy: a case control study on a clinical, neurophysiological and biochemical characteristics
}

Clarissa Troller Habekost ${ }^{1,11}$, Pedro Schestatsky ${ }^{5,7}$, Vitor Felix Torres ${ }^{7}$, Daniella Moura de Coelho ${ }^{6}$, Carmen Regla Vargas ${ }^{4,6}$, Vitor Torrez ${ }^{2}$, Jean Pierre Oses ${ }^{10,12}$, Luis Valmor Portela ${ }^{2,3,12}$, Fernanda dos Santos Pereira ${ }^{8}$, Ursula Matte ${ }^{1,9,11}$ and Laura Bannach Jardim 1,5,6,8,11*

\begin{abstract}
Background: Neurologic impairments in female heterozygotes for X-linked Adrenoleukodystrophy (X-ALD) are poorly understood. Our aims were to describe the neurological and neurophysiological manifestations of a cohort of X-ALD heterozygotes, and to correlate them with age, disease duration, mutations, $\mathrm{X}$-inactivation and serum concentrations of a marker of neuronal damage, neuron-specific enolase (NSE).

Methods: All 45 heterozygotes identified in our region, with previous VLCFA and molecular diagnosis, were invited to be evaluated through myelopathy scales JOA and SSPROM, nerve conduction studies and somatosensory evoked responses. X inactivation pattern was tested by HUMARA methylation assay. Serum NSE was measured by eletrochemiluminescense.
\end{abstract}

Results: Thirty three heterozygote women were recruited: 29 (87\%) were symptomatic. Symptomatic and asymptomatic women presented different $m \pm$ sd ages ( $43.9 \pm 10.2$ versus $24.3 \pm 4.6)$, JOA (14.5 \pm 1.7 versus $16.6 \pm 0.2)$ and SSPROM ( $86.6 \pm 7.9$ versus $98.4 \pm 1.1)$ scores $(p<0.05)$. Both JOA $(r=-0.68)$ and SSPROM $(r=-0.65)$ correlated with age, irrespectively of the disease status ( $p=0.0001$, Spearman). Delayed latencies in the central ascending conduction studies on the lower limbs were present in 72\% of all heterozygotes, and correlated with SSPROM $(r=-0.47, p=0.018$, Spearman). NSE values were higher in heterozygote than in control women $(12.9 \pm 7$ and $7.2 \pm 7 \mathrm{ng} / \mathrm{ml}, p=0.012$, Mann-Whitney U). Mutation severity and inactivation patterns were not associated with neurologic status.

Conclusion: Neurologic manifestations, clearly related to age, were quite common in the present cohort. JOA and SSPROM scales were able to discriminate the asymptomatic from the symptomatic heterozygotes. Both scales might be useful tools to follow disease progression, in future studies.

Keywords: X-linked adrenoleukodystrophy, X-ALD heterozygote females, X-ALD carriers, JOA, SSPROM, Neuron-specific enolase, Evoked potentials, Nerve conduction, X inactivation, Spastic paraplegia

\footnotetext{
* Correspondence: ljardim@hcpa.ufrgs.br

${ }^{1}$ Post-Graduation Program in Genetics and Molecular Biology, Universidade

Federal do Rio Grande do Sul (UFRGS), Porto Alegre, Brazil

Full list of author information is available at the end of the article
} 


\section{Background}

X-linked adrenoleukodystrophy (X-ALD, OMIM \#300100) is the most common peroxisomal disorder worldwide $[1,2]$. Affecting the metabolism of saturated very long chain fatty acids (VLCFAs), X-ALD is caused by a defect in $A B C D 1$ gene that codes for ALD protein [3], a peroxisomal membrane protein that belongs to the ATP-binding cassette superfamily of membrane transport proteins [4]. $A B C D 1$ mutations give rise to obvious phenotypes in hemizygote men, such as the classical cerebral form of ALD (CALD) and the adrenomyeloneuropathy (AMN), among others [4].

A very limited number of studies addressed manifestations in female carriers; some authors stated that affected women are under recognized and undertreated [5]. Early reports said that 20 to $50 \%$ of heterozygotes might present a mild to severe myeloneuropathy that resembles AMN, this clinical picture being related to ageing [6]. The myelopathic component of AMN is characterized by a distal axonopathy that affects the dorsal column and the corticospinal tract [7]. Neurophysiologic studies showed central and peripheral abnormalities and suggested the prominence of axonal dysfunction [8].

VLCFA were established a long time ago as biomarkers for X-ALD. Elevated plasma VLCFA levels are the diagnostic gold-standard for X-ALD in men and present $15-20 \%$ of false negative results in women, for whom a molecular investigation is the main diagnostic tool [4].

In addition to diagnostic concerns, there are no reasonable mechanistic explanations for the variability regarding the presence and absence of neurological impairments in heterozygote females. Although brain VLCFA levels correlated with CALD in hemizygotes [9], plasma VLCFA levels have never been related to $\mathrm{X}$-ALD phenotypes in men and women [4]. It is well known that genotypes do not correlate with phenotypes, in X-ALD [4]. Skewed X chromosome inactivation was also investigated, bringing contradictory results [10-12]. Alterations in oxidative stress parameters such as thiobarbituric acid-reactive substances [13] have been described in female carriers, without further exploring their potential associations with phenotypes.

Therefore, clinical and biochemical biomarkers of disease progression in heterozygotes for X-ALD are lacking. In this sense, we aimed to describe the symptomatic status of our X-ALD heterozygote cohort; to measure the neurological manifestations through myelopathy scales JOA and SSPROM, peripheral nerve conduction studies and somatosensory evoked responses; to measure plasma levels of a neuron disease marker, neuron-specific enolase (NSE); and to look for associations between these parameters and independent variables such as age, age at onset, mutations, $\mathrm{X}$ inactivation pattern and plasma VLCFA.

\section{Methods}

Population and clinical evaluations

All women previously identified as heterozygotes for $\mathrm{X}$-ALD, in South Brazil, were invited to participate in this study. All had molecular analyses and VLCFA dosages performed in our institution, as reported elsewhere [14]. To be included, they had to be over 18 years old and give the informed consent. Exclusion criteria were the presence of any abnormality on the following exams at recruitment: lymphocytes count, hemoglobin, erythrocytes median corpuscular volume, sedimentation rate, vitamin $\mathrm{B} 12$, thyroid stimulating hormone, proteinogram, VDRL (Venereal disease research laboratory), FTA-Abs (Fluorescent Treponemal Antibody- absorption), antibodies anti-HTLV (Human T lymphotropic virus) and Anti-HIV (Human Immunodeficiency Virus), oxalacetic and glutamic pyruvic transaminases, qualitative urine test, thrombocytes, glucose and creatine.

The interview started with an open question about any disability or presence of symptoms in the heterozygote. After that, the interviewer (CTH) described the symptoms of spasticity, paraparesis, sensory losses and loss of sphincter control, and explained that these symptoms could be present in heterozygote females. After that, the interviewer asked again about the presence and age at onset (AO) of motor disability, sensory losses and/or loss of sphincter control and the answer to this was taken into account. Heterozygote women were then classified according to their responses; as symptomatic if complaints of neuropathic pain, paresthesia, sphincter dysfunction or paresis were present, or as asymptomatic if these complaints were absent. Data such as age, AO, disease duration (DD), family and $\mathrm{ABCD} 1$ mutations were collected.

Neurological impairment and disability were evaluated through two myelopathy scales: the Japanese Orthopaedic Association (JOA) [15] and the Severity Score System for Progressive Myelopathy (SSPROM) [16]. JOA includes questions about motor disability of upper and lower limbs, about sensory losses and about sphincter function. SSPROM includes questions about these same domains, plus questions about motor strength and tonus/reflexes. In this sense, both scales measure disability as well as neurological impairment.

We elected two SSPROM domains to measure disability. SSPROM's motor disability domain was based on and mirrors the Overall Disability Sum Score [17], while SSPROM's sphincter domain was based and recapitulates the "sphincter dysfunction" domain of Kurtzke functional systems scores [18]. An overall disability evaluation was then measured by these two SSPROM domains (0 to 50 points; Table 1 ). Since a minimally important difference has not been determined for the disability parameters so far, we have 
Table 1 Clinical characteristics of the local cohort of X-ALD heterozygote women

\begin{tabular}{|c|c|c|c|c|}
\hline & \multicolumn{3}{|c|}{ X-ALD heterozygotes } & \multirow[t]{2}{*}{$\mathbf{p}^{*}$} \\
\hline & All heterozygotes participant & Symtomatic & Asymtomatic & \\
\hline N (families) & (16) 33 & 29 & 4 & \\
\hline \multirow[t]{2}{*}{ Age at examination } & $41.2 \pm 11.9$ & $43.9 \pm 10.2$ & $24.3 \pm 4.6$ & 0.005 \\
\hline & & $(24-61)$ & $(20-30)$ & \\
\hline Age at onset of symptoms & & $39.4 \pm 10(21-59)$ & & \\
\hline Disease duration & & $4.5 \pm 3.3$ & & \\
\hline JOA (range: -2 to 17 ) & $14.8 \pm 1.8$ & $14.5 \pm 1.7$ & $16.6 \pm 0.2$ & 0.005 \\
\hline SSPROM (range: 0 to 100) & $88 \pm 8.4$ & $86.6 \pm 7.9$ & $98.4 \pm 1.1$ & 0.001 \\
\hline SSPROM Motor Disability (range: 0 to 30 ) & $27.6 \pm 2.6$ & $27.3 \pm 2.6$ & $30 \pm 0$ & 0.023 \\
\hline SSPROM Motor Strength (0 to 20) & $18.9 \pm 2$ & $19.1 \pm 1.3$ & $17.5 \pm 5$ & ns \\
\hline SSPROM sensory losses (0 to 20) & $16.4 \pm 2.4$ & $16.1 \pm 2.3$ & $18.5 \pm 9$ & ns \\
\hline SSPROM spasticity/ hyperreflexia (0 to 10) & $6.1 \pm 2.1$ & $5.7 \pm 1.8$ & $9.4 \pm 1.2$ & 0.05 \\
\hline SSPROM sphincter contro (0 to 30 ) & $27.6 \pm 2.6$ & $17.8 \pm 2.3$ & $20 \pm 0$ & ns \\
\hline
\end{tabular}

arbitrated that women with an overall score $\leq 47$ points were already disabled.

In order to produce a "disease severity score" where the neurological impairment was corrected by the disease duration, the SSPROM reciprocate of each woman was divided by disease duration. This severity score was then compared among groups stratified by risk factors such as mutation position, mutation severity, NSE and inactivation patterns.

Blood samples were taken and neurophysiological studies (described below) were performed within 15 days of evaluation.

\section{Neurophysiological studies}

Motor and sensory nerve conduction studies in several nerves were performed in the right side of the body (Table 2). Motor conduction velocity (m-NCV), distal latency and amplitude for both compound muscle action potential (CMAP) and sensory nerve action potential (SNAP) were measured according to standard techniques in which normal values were considered within 2-SD from the mean [19].

Somatosensory evoked responses (SSER) were measured using the 10/20 electrode placement international system and recorded according to standard techniques [19]. The latencies of N8, P40 and N50 were recorded after tibial nerve stimulation, and N9 and N20 after median nerve stimulation. Only P40 and N20 latencies were included into regression analyses. Normal latency values were considered according to Chiappa [20] and based on the patient's height. The upper limits of normal values were given in Table 3.

Both, nerve conduction studies and SSER were performed using a Nihon -Kohden (Neuropack S1 MEB$9400 \mathrm{~K}) 4$ channel EMG/EP system.

\section{VLCFA}

Plasma docosanoic (C22:0), tetracosanoic (C24:0) and hexacosanoic $(\mathrm{C} 26: 0)$ acids were obtained previously to the present study, and were analyzed as described by others [21]. Values of C26:0 (in $\mu \mathrm{m} / \mathrm{L}$ ) and of discriminant factor 3,805(C24:0/C22:0) + 5,296(C26:0/C22:0) + $5,15(\mathrm{C} 26: 0)$ [22] were studied in the present heterozygote females. The discriminant factor's value of 10.86 discriminated X-ALD heterozygote from normal homozygote women, with $15 \%$ of false-negative results.

\section{Enolase quantification}

Serum NSE was measured using an eletrochemiluminescent assay provided by Roche DiagnosticsR, Indianapolis, IN, a double sandwich assay that uses an antibody antiNSE bound with ruthenium (luminescent label). The reaction and quantification were performed by Elecsys2010 (Roche). No hemolyzed samples were used. The assay was carried out in duplicate and the confidence variance was within 5\%. NSE concentrations were also measured in thirteen unrelated, normal women used as a control group.

\section{Inactivation studies}

Genomic DNA was obtained from peripheral leukocytes by the salting out procedure [23]. X-inactivation patterns were assessed for skewing with the human androgenreceptor locus (HUMARA) methylation assay [24]. Prior to digestion, informative women were identified by PCR at HUMARA locus and capillary electrophoresis. For the informative women $(n=20)$, digestion of $100 \mathrm{ng}$ genomic DNA with the methylation-sensitive restriction endonuclease, HpaII, and subsequent PCR amplification of the HUMARA polymorphic CAG repeat was used to determine $\mathrm{X}$-inactivation status in X-ALD heterozygotes. 
Table 2 Nerve conduction studies according to the presence or absence of symptoms of 22 -ALD heterozygote women

\begin{tabular}{|c|c|c|c|c|c|}
\hline \multicolumn{2}{|c|}{ Neuroconduction studies, right side (Mean \pm sd) (range) } & \multirow{2}{*}{$\begin{array}{c}\begin{array}{c}\text { All } 22 \\
\text { heterozygotes }\end{array} \\
57 \pm 6.2(47-73)\end{array}$} & \multicolumn{2}{|c|}{ Presence of symptoms } & \multirow[t]{2}{*}{$\mathbf{p}^{*}$} \\
\hline Motor conduction velocity (m/s) & Median & & $\begin{array}{c}\text { Symptomatic (20) } \\
56.9 \pm 6.5\end{array}$ & $\begin{array}{c}\text { Asymptomatic (2) } \\
58.9 \pm 2.2\end{array}$ & \\
\hline & (results above the cutoff value of 49,2 ) & & (2) & (0) & \\
\hline & Fibular & $48.1 \pm 5(40-55)$ & $47.3 \pm 4.9$ & $53.2 \pm 1$ & ns \\
\hline & (results above the cutoff value of 45.3) & & (3) & (0) & \\
\hline & Tibial & $49.5 \pm 4.6(44-63)$ & $49.2 \pm 4.6$ & 54.4 & ns \\
\hline & (results above the cutoff value of 39.9) & & (0) & (0) & \\
\hline \multirow[t]{6}{*}{ Sensitive conduction velocity (m/s) } & Median & $49.4 \pm 3.9(42-59)$ & $49.2 \pm 4$ & $52 \pm 2$ & ns \\
\hline & (results above the cutoff value of 51.7) & & $(12)$ & (0) & \\
\hline & Ulnar & $52.6 \pm 6(44-66)$ & $52.8 \pm 6.3$ & $51.3 \pm 0.6$ & ns \\
\hline & (results above the cutoff value of 53.8) & & (3) & (0) & \\
\hline & Fibular superficial & $53.7 \pm 5.7(44-64)$ & $53.1 \pm 5.4$ & $58.4 \pm 7.5$ & ns \\
\hline & (results above the cutoff value of 35) & & (0) & (0) & \\
\hline \multirow[t]{6}{*}{ Motor conduction amplitude (mV) } & Median & $9 \pm 3.6(2-16)$ & $9 \pm 3.7$ & $9.2 \pm 0.5$ & ns \\
\hline & (results under the cutoff value of 3.3) & & (1) & (0) & \\
\hline & Fibular & $4 \pm 1.9(1-7)$ & $4 \pm 1.8$ & $3.9 \pm 3.2$ & ns \\
\hline & (results under the cutoff value of 1.6) & & (1) & (0) & \\
\hline & Tibial & $7.7 \pm 4.7$ (2 to 19$)$ & $7.6 \pm 5$ & $8.1 \pm 2.3$ & ns \\
\hline & (results under the cutoff value of 2,8 ) & & (1) & (0) & \\
\hline \multirow[t]{6}{*}{ Sensitive conduction amplitude $(\mu \mathrm{V})$} & Median & $14.5 \pm 5.8(5$ to 29$)$ & $14.5 \pm 5.9$ & $15.2 \pm 7$ & ns \\
\hline & (results under the cutoff value of 15.1) & & (3) & (0) & \\
\hline & Ulnar & $11.3 \pm 6$ (5 to 27$)$ & $11.4 \pm 6.4$ & $10.9 \pm 3.3$ & ns \\
\hline & (results under the cutoff value of 5.8) & & (0) & (1) & \\
\hline & Fibular & $15.3 \pm 6.6(5$ a 30$)$ & $15.3 \pm 6.5$ & $15.4 \pm 11$ & ns \\
\hline & (results under the cutoff value of 10) & & $(2)$ & (1) & \\
\hline
\end{tabular}

*Mann-Whitney U.

The degree of chromosome $\mathrm{X}$ inactivation was calculated using the formula $[(\mathrm{d} 1 / \mathrm{u} 1) /(\mathrm{d} 1 / \mathrm{u} 1+\mathrm{d} 2 / \mathrm{u} 2)]$, where $\mathrm{d}=$ digested, $\mathrm{u}=$ undigested sample, and the two allele peaks (1 and 2) are compared [25]. Inactivation ratios less than 70:30 were considered random. Ratios greater than or equal to 70:30 and less than or equal to 90:10 were considered moderately skewed. Ratios greater than 90:10 were considered highly skewed [26].

\section{Statistical analyses}

Patient characteristics are given as mean \pm SD and range, when applicable. Categorical variables were represented by absolute and relative frequencies and were compared through Fisher exact test. The majority of continuous variables did not show a normal distribution on the Shapiro-Wilk test, and therefore were tested by MannWhitney U, Spearman correlation and, when applicable,

Table 3 Somatosensory evoked responses according to the presence or absence of symptoms of $25 \times$-ALD heterozygote women

\begin{tabular}{|c|c|c|c|c|c|}
\hline \multirow[t]{2}{*}{ Somatosensory evoked responses } & & \multirow{2}{*}{$\begin{array}{c}\text { All } 25 \\
\text { heterozygote } \\
\text { females }\end{array}$} & \multicolumn{2}{|c|}{ Presence of symptoms } & \multirow[t]{2}{*}{$p$} \\
\hline & & & $\begin{array}{l}\text { Symptomatic } \\
\text { women: } 23\end{array}$ & $\begin{array}{l}\text { Asymtomatic } \\
\text { women: } 2\end{array}$ & \\
\hline \multirow{2}{*}{$\begin{array}{l}\text { Upper Limbs (N20, or somatosensory cortex } \\
\text { contralateral to the wrist stimulated) in ms }\end{array}$} & Mean \pm sd & $19.2 \pm 1.1$ & $20.1 \pm 4.2$ & $18 \pm 0.9$ & $\mathrm{~ns}^{*}$ \\
\hline & Above the cutoff value of $20.5 \mathrm{~ms}$ & $6 / 25$ & $6 / 23$ & $0 / 2$ & ns \# \\
\hline \multirow{2}{*}{$\begin{array}{l}\text { Lower Limbs (P40, or somatosensory cortex } \\
\text { ipsilateral to the tibial nerve stimulated) in ms }\end{array}$} & Mean $\pm s d$ & $38 \pm 2,5$ & $55.3 \pm 32.8$ & $38.9 \pm 3.8$ & $\mathrm{~ns}$ * \\
\hline & Above the cutoff value of $41,5 \mathrm{~ms}$ & $18 / 25$ & $17 / 23$ & $1 / 2$ & ns \# \\
\hline
\end{tabular}

* Mann-Whitney $\mathrm{U} ; \mathrm{m} / \mathrm{s}=$ meters per second; $\mathrm{mV}$ - milivolts; $\mu \mathrm{V}=$ microvolts. 
by stepwise linear regression. Statistical significance was defined as $\mathrm{p}<0.05$. All statistical tests were performed in PASW 18.3.

\section{Results}

\section{Clinical findings}

Forty five heterozygote women (belonging to 25 families) had been detected by molecular studies in our institution, from 2008 to 2013. Thirty three heterozygotes (or $73 \%$ of the total heterozygotes detected in our region) gave their consent and were included in this study. The another 27\% (12 women) were lost: six were not found by two phone calls and letter, two refused to participate in the study and another four fulfilled exclusion criteria. The mean \pm sd ages of the 33 participants and of the 12 non-participants, in 2013, were of $41.2 \pm 11.9$ and $40.9 \pm$ 15 , respectively (ns, t test). Twenty-nine of the 33 participants classified themselves as symptomatic and four, as asymptomatic, after learning about X-ALD symptoms in females. Their general characteristics are present in Table 1.

Symptomatic were significantly older and presented worse JOA and SSPROM scores and higher disability scores than asymptomatic women. Significant differences in SSPROM scores were due to a high proportion of symptomatic females (27/29) with spasticity/hyperreflexia; and to motor disabilities (Table 1). The autoclassification and the disability scores did not perfectly match: normal scores in the overall disability evaluation were obtained in all four asymptomatic but also in 15/29 symptomatic women. Overall disability was related to SSPROM and JOA scores, but not to age, age at onset of symptoms, neurophysiologic studies and other biomarkers (data not shown).

Among symptomatic women, although SSPROM correlated with age and DD, only DD was maintained on linear regression $(\mathrm{r}=-0.73, \mathrm{p}=0.0001$, Spearman; $\mathrm{B}=-1.91$, $E P=0.28)$. When all heterozygote women were analyzed together, the association with age was confirmed $(\mathrm{r}=-0.65, \mathrm{p}=0.0001$, Spearman; $\mathrm{B}=-0.43, \mathrm{EP}=0,10$, by Linear regression) (Figures $1 \mathrm{~A}$ and $1 \mathrm{C}$ ).

Similar associations were found regarding to JOA. Among symptomatic women, although JOA scores were at first correlated with age, AO and DD, only DD was significantly related to JOA on stepwise linear regression $(\mathrm{r}=-0.61, \mathrm{p}<0.001$, Spearman; $\mathrm{B}=-0.37 ; \mathrm{EP}=0.07)$.

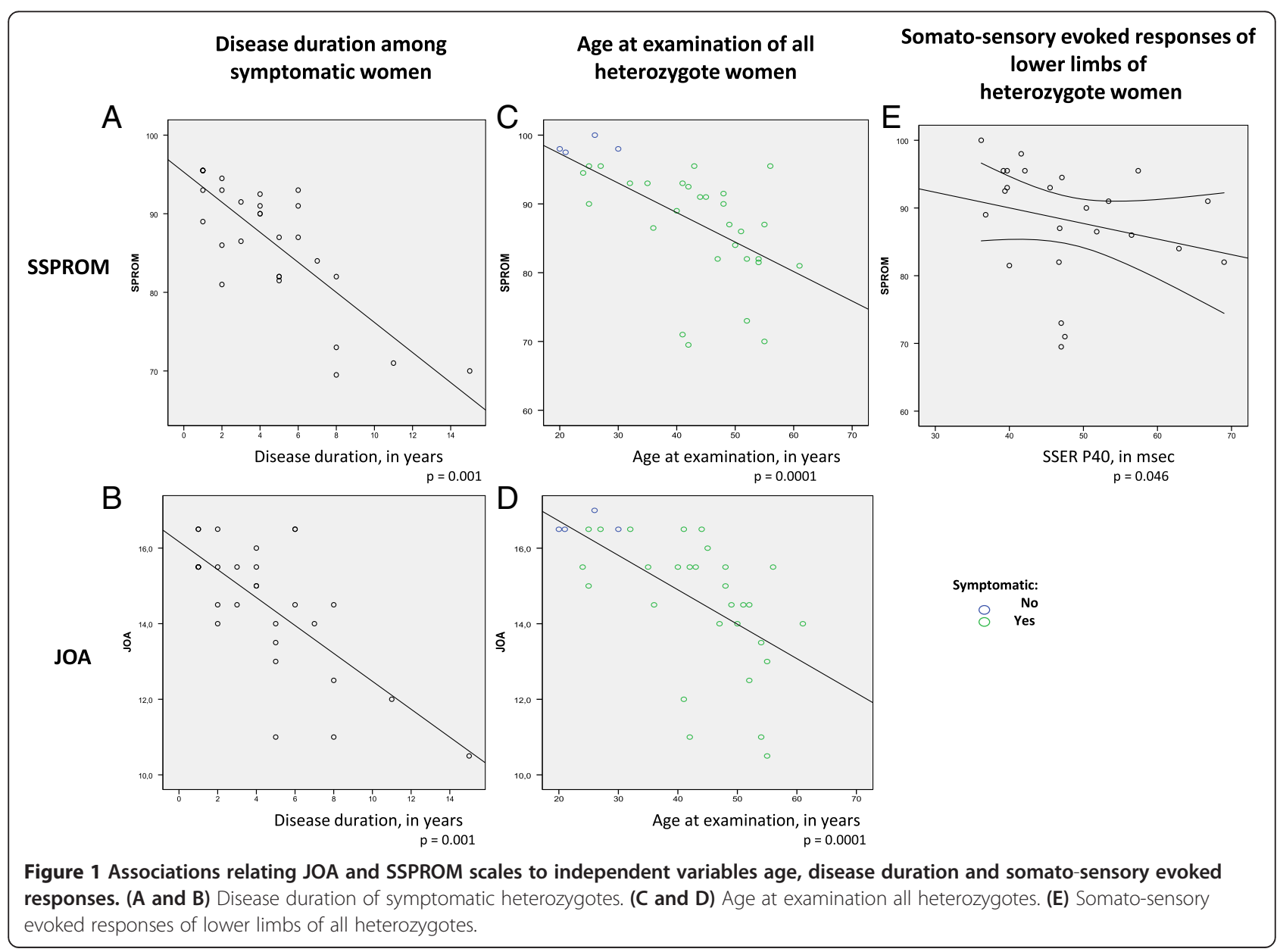


When all heterozygotes were analyzed together, JOA correlated with age $(\mathrm{r}=-0.68, \mathrm{p}=0.0001$, Spearman; $\mathrm{B}=-0.09 ; \mathrm{EP}=0.02$, Linear regression) (Figures $1 \mathrm{~B}$ and $1 \mathrm{D})$.

Mean \pm sd of disease severity, or $1 /($ SSPROM x DD), among symptomatic women, were $3.7 \pm 2.6$. This score did not correlate with age or any other parameters.

Symptomatic classification produced more associations with independent parameters than the disability classification, in this cohort. Due to that, it was maintained as the stratification criteria between the present heterozygote women, when necessary.

\section{Nerve conduction studies}

Twenty-two out of 33 heterozygotes (20 symptomatic and 2 asymptomatic women) performed neurophysiologic studies. The others denied for personal reasons. Nineteen (including one asymptomatic heterozygote) presented at least one altered proof. Results are presented in Table 2.

All $\mathrm{m}-\mathrm{NCV}$ and most of s-NCV were normal, and there were no correlations between NCVs and symptomatic status, age, AO, DD, disability status, JOA, SSPROM, disease severity, inactivation studies or plasma NSE concentrations (data not shown). Twelve out of 20 symptomatic heterozygotes showed reduced s-NCV of the median nerve, measured on the elbow. This finding was unrelated to any variable under study (data not shown).

No evidence of generalized axonal damage was found in any of the women studied, since the majority of CMAPs and SNAPs amplitudes were between the normal range. The amplitude of fibular superficial nerve SNAPs correlated with AO among the heterozygotes $(\mathrm{r}=0.55, \mathrm{p}=$ 0.015 , Spearman).

\section{Somatosensory evoked responses}

Twenty-five heterozygote females (including those $22 \mathrm{fe}$ males with nerve studies) were tested for SSE. Eighteen (17 symptomatic) presented delayed latencies in the central ascending conduction studies starting on the lower limbs (evoked potential P40) (Table 3). Latencies of the evoked potentials were not related to age, disability, disease severity or to the symptomatic status of the heterozygote women (ns, Spearman and Mann-Whitney U tests). SSPROM correlated weakly with lower limbs evoked potential latencies $(r=-0.47, p=0.018$, Spearman, Figure 1E).

\section{Mutations found in $A B C D 1$ gene}

The mutations detected on $A B C D 1$ gene are depicted on Additional file 1: Table S1. These mutations were stratified according to their exonic position in the gene as well as divided according to their severity in missense or nonsense/frameshift. Mutation severity or position did not correlate either to age at onset of symptoms or to disease severity (Mann-Whitney U and Kruskal-Wallis, data not shown).

\section{$\mathrm{X}$ Chromosome inactivation patterns}

Twenty heterozygotes were informative in the technique applied to determine the inactivation pattern. Six had a skewed inactivation pattern of 70:30 or more. Skewed inactivation was not associated to symptomatic state (ns, Fisher exact test), disability, SSPROM, JOA, disease severity (ns, Spearman) or AO (ns, Linear Regression). A curious trend to relate larger skewing with age was also seen $(\mathrm{r}=0.37, \mathrm{p}=0.1$, Spearman).

\section{NSE}

The medium $\pm \mathrm{sd}$ values of NSE were higher in heterozygotes than in controls (12.9 \pm 7 versus $7.2 \pm 7 \mathrm{ng} / \mathrm{ml}$, $\mathrm{p}=0.012$, Mann-Whitney U, Figure 2). Both heterozygote and control groups had similar ages (41.2 \pm 11.9 and $35.5 \pm 10.4$ years, ns, M-W U).

NSE was not correlated with age, either among all women or just among heterozygotes. No correlations were found between NSE and AO, disability or symptomatic status, inactivation studies, position of mutation in ABCD1 gene, SSPROM, JOA, disease severity, evoked responses or other neurophysiology parameters (data not shown).

\section{VLCFA}

VLCFA had been determined at the time of diagnosis of the present 33 heterozygotes, sometimes several years before the present evaluation. Their mean \pm sd ages at the collection were of $30 \pm 11.3$ (range: 7 to 43 ) yearsold. In the case of symptomatic women, for whom the symptom onset has been determined as year zero, blood collections were done at a $-3.8 \pm 4.5$ (range: -12 to 3 ) years from the start of their symptoms. Correlations of VLCFA levels with age at the time of blood collection and with time from disease start were then tested. There were direct correlations between age and both C26:0 and VLCFA discriminant factor $(\mathrm{r}=0.58, \mathrm{p}=0.011$ and $r=0.60, p=0.007$, Spearman, Additional file 2: Figure S1).

\section{Discussion}

We examined $73 \%$ of all known heterozygote women for $\mathrm{X}$-ALD followed in our institution, and detected significant clinical and neurophysiologic impairments in $87 \%$ of them. The clinical scales JOA and SSPROM were able to differentiate symptomatic from asymptomatic heterozygotes. Both scales were associated with DD of the symptomatic cases and with age in the overall group. Abnormalities in somatosensory evoked responses, mostly from the lower limbs, were quite frequent. A plasma protein related to neuronal damage, NSE, was associated with the heterozygote state. All these might be candidates as 


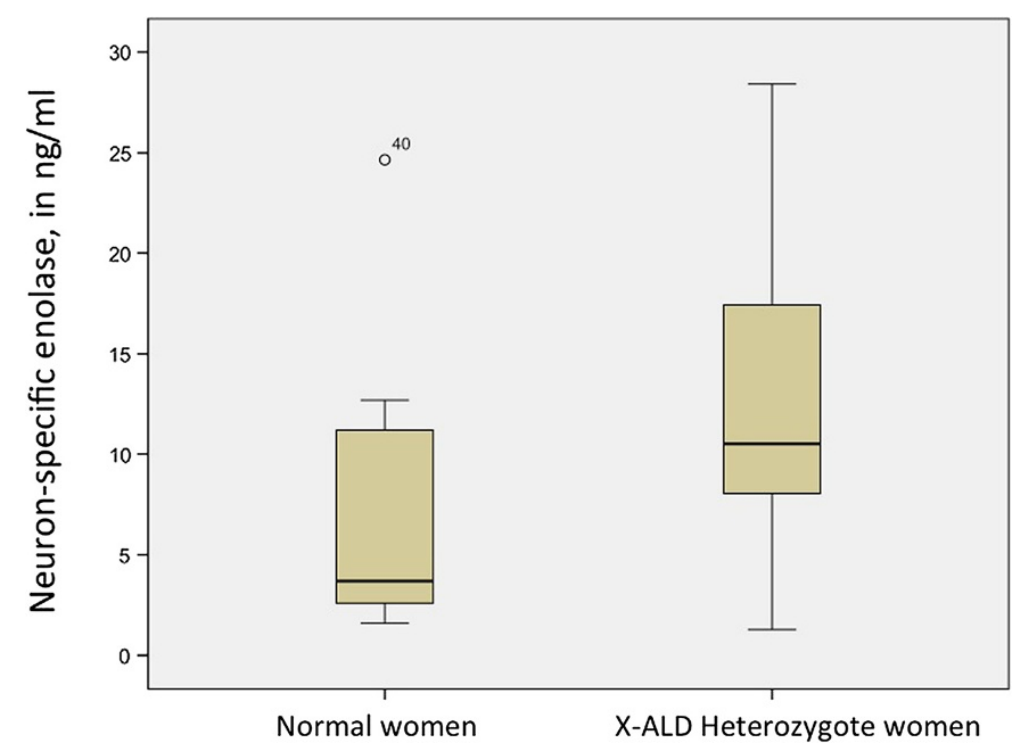

Figure 2 NSE among the X-ALD heterozygotes and the control women.

biomarkers for disease progression in heterozygotes for X-ALD.

Quantifying physical disability and neurologic burden is essential to understanding the progression rate of any disease [5]. In the present report, three levels of consequences related to the heterozygote state for X-ALD were measured: subjective perception, neurologic impairment and disability. Being symptomatic is a yes/or/ no subjective perception. Neurologic impairment was measured by JOA and SSPROM scales, and by neurophysiologic studies. Disability reflects dysfunctions on performance and activity; we measured it by using specific domains of SSPROM. Disability needs an arbitrary cutoff. Disability scores obtained in the present group were very mild. Due to that, we decided to include even small disabilities in the dichotomic, positive group. We have then stratified heterozygote women both by their symptomatic and disability states.

We have obtained 29 symptomatic and 4 asymptomatic women; in contrast, there were 14 disabled and 19 non-disabled women. Although the disability stratification seemed to produce more even groups, it failed to be associated with age or with DD. In contrast, symptomatic status correlated with age, JOA, and SSPROM; moreover, DD - in other words, the duration of symptoms - correlated with both JOA and SSPROM. Therefore, symptomatic classification produced more useful information than its counterpart (disability).

Neurological impairment was better evaluated by clinical scales than by neurophysiology. Both JOA and SSPROM scales were able to discriminate symptomatic from asymptomatic heterozygotes, with almost no overlap, correlated very well with DD in the symptomatic group, and with age in the overall group.

The lack of any substantial alterations on peripheral neurophysiology reported here corroborates the findings of Schmidt and Cols [8]. One interesting finding was the direct correlation of fibular SNAP with AO. Reduced SNAP is commonly related to an inherent susceptibility of the fibular nerve for entrapments. Why did it correlate with $\mathrm{AO}$, is an issue that deserves investigation in other heterozygotes.

After clinical scores, the SSER of lower limbs have shown the most prevalent abnormalities in heterozygote women. Similar results have been seen by others [8,27-30] SSER of lower limbs correlated with SSPROM and this might be taken as an external validation for SSPROM usefulness among X-ALD heterozygotes. On the other hand, SSER were not associated with any other measurement of disease progression like age and disease duration.

$\mathrm{X}$ chromosome inactivation has been studied as a modifying factor that could influence clinical presentation in women with X linked disorders. Relating a skewed inactivation in peripheral leukocytes to a neuronal disorder, as in the heterozygote women with X-ALD, is a complex hypothesis. If this association would exist, at least two possible mechanisms should be invoked: a good mirroring between neurons and leukocytes, where a skewed $\mathrm{X}$ inactivation in neurons would be related to differences in clinical presentation; and/or an intercellular mechanism, operating from skewed cells other than neurons. Differently from some authors [12] but similar to others [11], we did not find any association between inactivation and 
symptomatic status or neurological scores. We observed a trend relating an increased skewing with age, which is in accordance with literature [31].

VLCFA also increased with age, at least in this cohort, whose blood collections had been done between 7 and 43 years of age. Other authors have observed a similar finding in this age group, with decreasing VLCFA concentrations in women older than 50 years old [32]. Although both VLFCA and skewing inactivation increased with age in our group, no association was found with increasing neurological symptoms.

NSE is a cytoplasmatic glycolytic enzyme found in neurons and cells with neuroendocrine differentiation. Since NSE is not physiologically secreted, an increase of its serum concentrations can be associated with structural damage to neuronal cells [33]. NSE concentrations were higher in heterozygote women than in controls, in the present study. Since NSE was not associated with symptomatic or disability status, nor with any variable related to disease progression, we were not able to show a clear potential for NSE as a biomarker for disease progression in this disease.

According to former studies, around $50 \%$ of X-ALD heterozygotes showed some degree of neurologic involvement [6]. Our results were quite different: $87 \%$ were symptomatic, with neurological impairments as measured by JOA and SSPROM scores. Our large proportion of symptomatic women was quite surprising. Although one cannot rule out a selection bias favoring the recruitment of symptomatic heterozygotes, the similitude between the mean ages of the recruited and of the non-recruited heterozygote women works against this conjecture. Our rates might be explained by different measurements of neurological burden: perhaps former reports relied on disabilities rather than impairments. Our experience showed that disabilities were mild in general, with the exception of sphincter dysfunctions (Table 1). Impairments, on the other hand, were clearly present, the major impact being the corticospinal involvement (Table 1).

Emotional issues should also be remembered. Male $\mathrm{X}$-ALD is a catastrophic disease for families. A mother or a female caregiver of an affected boy usually lives in very stressful conditions, where priorities are put in hemizygote men rather than in her well-being. Maybe the actual numbers of symptomatic heterozygote females had been previously underestimated, due to a hasty report of these women about their healthy states. The hypothesis that the majority of heterozygote females will present a neurologic impairment is not negligible. Moreover, the associations between JOA and SSPROM with age might suggest that the symptomatic status is a matter of time and that, as heterozygote females grow older, they get symptomatic.
With the prominent involvement of pyramidal tracts, $\mathrm{X}$-ALD presenting in symptomatic heterozygotes and as male AMN, can be seen as one form of complicated spastic paraplegia (SPG) [34]. Therapeutic trials in men and women with AMN and in SPGs in general are expected in a near future, and it will be important to decide what scales to use on them. Recently, some studies have measured the neurological impairment of SPGs by the Spastic Paraplegia Rating Scale (SPRS) [35]. In contrast, we have used two instruments fitted to measure both disability and impairment, scales that might be called disease severity scoring systems (or 3S): the scales JOA and SSPROM. SSPROM was developed to follow metabolic progressive myelopathies such as AMN; so far, only the present and the description studies have used it [16]. JOA was developed to follow cervical compression myelopathies [15], and it is probably the most used neurologic scale for myelopathies so far. For a clinical trial, several properties of the chosen scale will be essential to guarantee the best design, such as validity, reliability and sensitivity to change. At this very moment, it is not clear what scale will be best fitted to a future trial for AMN. Comparisons of these characteristics among different scales are needed and should be done in a near future.

\section{Conclusions}

Neurologic impairments are frequent and are clearly related to age and to the CNS involvement, in heterozygote females. SSER of lower limbs and plasma NSE concentrations were altered in the majority of heterozygote females. Since they did not differentiate between symptomatic and asymptomatic heterozygotes, nor were related to age or disease duration, we cannot postulate them as good candidates as biomarkers of disease progression. VLFCA and skewing inactivation were also unable to separate symptomatic from asymptomatic heterozygotes. Both increased with age, but dissociated from the increasing neurological symptoms. Among the number of potential biomarkers of disease progression, JOA and SSPROM presented the most robust associations with disease duration and age. Besides that, SSPROM correlated with SSER of lower limbs. We suggest that JOA and SSPROM might be valuable instruments in future natural history studies in heterozygotes for X-ALD.

\section{Additional files}

Additional file 1: Table S1. Mutations found at ABCD1 gene in the present X-ALD heterozygote women, and the distribution of ages at examination and at onset of symptoms.

Additional file 2: Figure S1. Associations between age and (6A) C26:00 plasma levels and (6B) VLCFA discriminant factor 3,805(C24:0/C22:0) + 5,296(C26:0/C22:0) + 5,15(C26:0). 


\section{Abbreviations}

AMN: Adrenomyeloneuropathy; AO: Age at onset; C22:0: Docosanoic acid; C24:0: Tetracosanoic acid; C26:0: Hexacosanoic acid; CALD: Cerebral form of ALD; CMAP: Compound muscle action potential; DD: Disease duration; HUMARA: Human androgen-receptor locus; JOA: Japanese Orthopaedic Association scale of myelopathy; m-NCV: Motor conduction velocity; NSE: Neuron-specific enolase; SNAP: Sensory nerve action potential; SPG: Spastic paraplegia; SPRS: Spastic Paraplegia Rating Scale;

SSER: Somatosensory evoked responses; SSPROM: Severity Score System for Progressive Myelopathy; VLCFA: Very long chain fatty acids; X-ALD: X-linked Adrenoleukodystrophy.

\section{Competing interests}

The authors declare that they have no competing interests.

\section{Authors' contributions}

$\mathrm{CTH}$ conceived the study, participated in the design of the study, carried out the recruitment, interviews and clinical studies, and helped to draft the manuscript. PS carried out the SSER studies and participated in the analysis. PS and VTF carried out peripheral neurophysiology studies and participated in the analysis of the data. DMC performed VLCFA analyses and helped in the coordination of data. CRV performed VLCFA analyses and helped in the acquisition of funding. VT performed NSE analyses and participated in the analysis of data. LVP performed NSE studies and helped in the acquisition of funding. FSP carried out the molecular genetic studies and participated in the analysis. UM participated in the molecular analysis and in the coordination of data. LBJ conceived the study, participated in the design and coordination of the study, performed the statistical analysis, and drafted the manuscript. All authors read and approved the final manuscript.

\section{Acknowledgements}

We would like to thank the patients and their families for taking part in this study. We would also like to thank Raphael M. Castilhos and Deborah Blank for their technical assistance and contribution in early stages of this project; Karina C. Donis, André dos Anjos and Jonas Alex Saute for their contribution in recruiting some patients; and Ana Louzada for her technical assistance in the neurophysiological studies. This work was supported by Fundo de Incentivo à Pesquisa do Hospital de Clínicas de Porto Alegre (FIPE-HCPA), Project GPPG HCPA 110308; and by Instituto Nacional de Ciência e Tecnologia em Excitotoxicidade e Neuroproteção (INCTEN), Porto Alegre, Brazil. CTH were funded by INAGEMP - Instituto Nacional de Genética Médica Populacional. Pereira FS, Matte U, Portela LV and Jardim LB are funded by CNPq - Conselho Nacional de Desenvolvimento Científico e Tecnológico.

\section{Author details}

${ }^{1}$ Post-Graduation Program in Genetics and Molecular Biology, Universidade Federal do Rio Grande do Sul (UFRGS), Porto Alegre, Brazil. ${ }^{2}$ Post-Graduation Program in Biochemistry, Universidade Federal do Rio Grande do Sul (UFRGS), Porto Alegre, Brazil. ${ }^{3}$ Department of Biochemistry, Universidade Federal do Rio Grande do Sul (UFRGS), Porto Alegre, Brazil. ${ }^{4}$ Department of Analysis, Universidade Federal do Rio Grande do Sul (UFRGS), Porto Alegre, Brazil. ${ }^{5}$ Department of Internal Medicine, Universidade Federal do Rio Grande do Sul (UFRGS), Porto Alegre, Brazil. ${ }^{6}$ Medical Genetics Service, Hospital de Clínicas de Porto Alegre, Porto Alegre, Rio Grande do Sul, Brazil. ${ }^{7}$ Neurology Service, Hospital de Clínicas de Porto Alegre, Porto Alegre, Rio Grande do Sul, Brazil. ${ }^{\circ}$ Laboratory of Genetic Identification, Hospital de Clínicas de Porto Alegre, Porto Alegre, Rio Grande do Sul, Brazil. ' ${ }^{2}$ Laboratory of Gene Therapy, Hospital de Clínicas de Porto Alegre, Porto Alegre, Rio Grande do Sul, Brazil. ${ }^{10}$ Laboratório de Neurociências Clínicas, Centro de Ciências da Vida e da Saúde, Universidade Católica de Pelotas, Pelotas, Brazil. ${ }^{11}$ Instituto Nacional de Genética Médica Populacional (INAGEMP), Porto Alegre, Brazil. ${ }^{12}$ Instituto Nacional de Ciência e Tecnologia em Excitotoxicidade e Neuroproteção (INCTEN), Porto Alegre, Brazil.

Received: 2 December 2013 Accepted: 3 January 2014

Published: 13 January 2014

\section{References}

1. Bezman L, Moser AB, Raymond GV, Rinaldo P, Watkins PA, Smith KD, Kass $\mathrm{NE}$, Moser HW: Adrenoleukodystrophy: incidence, new mutation rate, and results of extended family screening. Ann Neurol 2001, 49(4):512-517.
2. Dubois-Dalcq M, Feigenbaum V, Aubourg P: The neurobiology of X-linked adrenoleukodystrophy, a demyelinating peroxisomal disorder. Trends Neurosci 1999, 22(1):4-12. Review.

3. Mosser J, Lutz Y, Stoeckel ME, Sarde CO, Kretz C, Douar AM, Lopez J, Aubourg P, Mandel $\mathrm{J}$ : The gene responsible for adrenoleukodystrophy encodes a peroxisomal membrane protein. Hum Mol Genet 1994, 3(2):265-271.

4. Moser H, Smith K, Watkins P, Powers J, Moser A: X-linked adrenoleukodystrophy. In The metabolic and molecular bases of inherited disease. Edited by Scriver C, Beaudet A, Sly W, Valle D. New York, N.Y: McGraw-Hill; 2001:3257-3301.

5. Jangouk P, Zackowski KM, Naidu S, Raymond GV: Adrenoleukodystrophy in female heterozygotes: underrecognized and undertreated. Mol Genet Metab 2012, 105(2):180-185.

6. Moser HW, Moser AB, Naidu S, Bergin A: Clinical aspects of adrenoleukodystrophy and adrenomyeloneuropathy. Dev Neurosci 1991, 13:254-261.

7. Zackowski KM, Dubey P, Raymond GV, Mori S, Bastian AJ, Moser HW: Sensorimotor function and axonal integrity in adrenomyeloneuropathy. Arch Neurol 2006, 63:74-80.

8. Schmidt S, Träber F, Block W, Keller E, Pohl C, von Oertzen J, Schild H, Schlegel $U$, Klockgether T: Phenotype assignment in symptomatic female carriers of X-linked adrenoleukodystrophy. J Neurol 2001, 248(1):36-44.

9. Asheuer M, Bieche I, Laurendeau I, Moser A, Hainque B, Vidaud M, Aubourg $P$ : Decreased expression of ABCD4 and BG1 genes early in the pathogenesis of X-linked adrenoleukodystrophy. Hum Mol Genet 2005, 14(10):1293-1303. Epub 2005 Mar 30.

10. Migeon BR, Moser HW, Moser AB, Axelman J, Sillence D, Norum RA: Adrenoleukodystrophy: evidence for $\mathrm{X}$ linkage, inactivation, and selection favoring the mutant allele in heterozygous cells. Proc Natl Acad Sci U S A 1981, 78(8):5066-5070.

11. Watkiss $E$, Webb T, Bundey S: Is skewed $X$ inactivation responsible for symptoms in female carriers for adrenoleucodystrophy? J Med Genet 1993, 30(8):651-654.

12. Maier EM, Muntau AC KS, Wichers M, Braun A, Roscher AA: Symptoms in carriers of adrenoleukodystrophy relate to skewed $\mathrm{X}$ inactivation, Ann. Neurol 2002, 52:683-688.

13. Deon M, Sitta A, Barschak AG, Coelho DM, Terroso T, Schmitt GO, Wanderley HY, Jardim LB, Giugliani R, Wajner M, Vargas CR: Oxidative stress is induced in female carriers of X-linked adrenoleukodystrophy. J Neurol Sci 2008, 266(1-2):79-83.

14. Pereira FS, Matte U, Habekost $C T$, de Castilhos RM, El Husny AS, Lourenço CM, Vianna-Morgante AM, Giuliani L, Galera MF, Honjo R, Kim CA, Politei J, Vargas CR, Jardim LB: Mutations, clinical findings and survival estimates in South American patients with X-linked adrenoleukodystrophy. PLOS One 2012, 7(3):e34195.

15. Yanenobu K, Abumi K, Negata K, et al: Interobserver and Intraobserver Reliability of the Japanese Orthopaedic Association Scoring System for Evaluation of Cervical Compression Myelopathy. Spine 2001, 26:1890-1895.

16. Castilhos RM, Blank D, Netto CB, Souza CF, Fernandes LN, Schwartz IV, Giugliani R, Jardim LB: Severity score system for progressive myelopathy: development and validation of a new clinical scale. Braz J Med Biol Res 2012, 45(7):565-572.

17. Merkies ISJ, Schmitz PIM, van der Meché FGA, et al: Clinimetric evaluation of a new overall disability scale in immune mediated polyneuropathies. J Neurol Neurosurg Psychiatry 2002, 72:596-601.

18. Kurtzke JF: Rating neurologic impairment in multiple sclerosis: An expanded disability status scale (EDSS). Neurology 1983, 33:1444-1452.

19. Dumitru D, Amato AA: Acquired neuropathies. In Electrodiagnostic Medicine. 2nd edition. Edited by Dumitru D, Zwarts MJ, Amato A. Philadelphia: Hanley and Belfus Inc; 2002:937-1041.

20. Chiappa KH: Evoked Potentials in Clinical Medicine. New York: Raven Press; 1997.

21. Moser HW, Moser AB: Measurement of saturated very long chain fatty acid in plasma. In Techniques of diagnostic human biochemical genetics. Edited by Hommes FA. New York: Wiley; 1991.

22. Moser AB, Kreiter N, Bezman L, Lu S, Raymond GV, Naidu S, Moser HW: Plasma very long chain fatty acids in 3,000 peroxisome disease patients and 29,000 controls. Ann Neurol 1999, 45(1):100-110.

23. Miller SA, Dykes DD, Polesky HF: A simple salting out procedure for extracting DNA from human nucleated cells. Nucleic Acids Res 1988, 16(3):1215. 
24. Allen RC, Zoghbi HY, Moseley AB, Rosenblatt HM, Belmont JW: Methylation of Hpall and Hhal sites near the polymorphic CAG repeat in the human androgen-receptor gene correlates with $\mathrm{X}$ chromosome inactivation. Am J Hum Genet 1992, 51(6):1229-1239.

25. Sharp A, Robinson D, Jacobs P: Age- and tissue-specific variation of $X$ chromosome inactivation ratios in normal women. Hum Genet 2000, 107:343-349.

26. Landgraf JMA, Cottle A, Plenge RM, Friez M, Schwatrz CE, Longshore J, Willard HF: X Chromosome-Inactivation Patterns of 1,005 Phenotypically Unaffected Females. Am. J. Hum. Gent 2006, 79:493-499.

27. Moloney JB, Masterson JG: Detection of adrenoleucodystrophy carriers by means of evoked potentials. Lancet 1982, 2:852-853.

28. Garg BP, Markand ON, DeMyer WE, Warren C Jr: Evoked response studies in patients with adrenoleukodystrophy and heterozygous relatives. Arch Neurol 1983, 40:356-359.

29. Shimizu H, Moser HW, Naidu S: Auditory brainstem response and audiologic findings in adrenoleukodystrophy: its variant and carrier. Otolaryngol Head Neck Surg 1988, 98:215-220.

30. Restuccia D, Di Lazzaro V, Valeriani M, Oliviero A, Le Pera D, Colosimo C, Burdi N, Cappa M, Bertini E, Di Biase A, Tonali P: Neurophysiological abnormalities in adrenoleukodystrophy carriers. Evidence of different degrees of central nervous system involvement, Brain 1997, 120(7):1139-1148.

31. Mengel-From J, Thinggaard M, Christiansen L, Vaupel JW, Orstavik KH, Christensen K: Skewed X inactivation and survival: a 13-year follow-up study of elderly twins and singletons. Eur J Hum Genet 2012, 20(3):361-364.

32. Stradomska TJ, Tylki-Szymańska A: Decreasing serum VLCFA levels in ageing X-ALD female carriers. J Inherit Metab Dis 2001, 24(8):851-857.

33. Tort AB, Portela LV, Rockenbach IC, Monte TL, Pereira ML, Souza DO, Rieder CR, Jardim LB: S100B and NSE serum concentrations in Machado Joseph disease. Clin Chim Acta 2005, 351(1-2):143-148.

34. Finsterer J, Löscher W, Quasthoff S, Wanschitz J, Auer-Grumbach M, Stevanin G: Hereditary spastic paraplegias with autosomal dominant, recessive, X-linked, or maternal trait of inheritance. J Neurol Sci 2012, 318(1-2):1-18.

35. Schüle R, Holland-Letz T, Klimpe S, Kassubek J, Klopstock T, Mall V, Otto S, Winner B, Schöls L: The Spastic Paraplegia Rating Scale (SPRS): a reliable and valid measure of disease severity. Neurology 2006, 67(3):430-434.

doi:10.1186/1750-1172-9-6

Cite this article as: Habekost et al:: Neurological impairment among heterozygote women for X-linked Adrenoleukodystrophy: a case control study on a clinical, neurophysiological and biochemical characteristics.

Orphanet Journal of Rare Diseases 2014 9:6.

\section{Submit your next manuscript to BioMed Central and take full advantage of:}

- Convenient online submission

- Thorough peer review

- No space constraints or color figure charges

- Immediate publication on acceptance

- Inclusion in PubMed, CAS, Scopus and Google Scholar

- Research which is freely available for redistribution 